typeset using JPSJ.sty $<$ ver.0.8 $>$

\title{
Antiferromagnetic Phases of One-Dimensional Quarter-Filled Organic Conductors
}

\author{
Hitoshi SEo and Hidetoshi FukuYAma \\ Department of Physics, Faculty of Science, University of Tokyo, Tokyo 113
}

(Received August 1, 2018)

\begin{abstract}
The magnetic structure of antiferromagnetically ordered phases of quasi-one-dimensional organic conductors is studied theoretically at absolute zero based on the mean field approximation to the quarter-filled band with on-site and nearest-neighbor Coulomb interaction. The differences in magnetic properties between the antiferromagnetic phase of (TMTTF) ${ }_{2} \mathrm{X}$ and the spin density wave phase in (TMTSF) $)_{2} \mathrm{X}$ are seen to be due to a varying degrees of roles played by the on-site Coulomb interaction. The nearest-neighbor Coulomb interaction introduces charge disproportionation, which has the same spatial periodicity as the Wigner crystal, accompanied by a modified antiferromagnetic phase. This is in accordance with the results of experiments by Nakamura et al on (TMTTF $)_{2} \mathrm{Br}$ and $(\mathrm{TMTTF})_{2} \mathrm{SCN}$. Moreover, the antiferromagnetic phase of (DI-DCNQI $)_{2} \mathrm{Ag}$ is predicted to have a similar antiferromagnetic spin structure.
\end{abstract}

KEYWORDS: TMTCF, nearest-neighbor Coulomb interaction, mean field approximation, antiferromagnetic spin ordering, charge disproportionation, DCNQI

Quasi-one-dimensional organic conductors which have a quarter-filled (or 3/4-filled) band such as $(\mathrm{TMTCF})_{2} \mathrm{X},(\mathrm{DCNQI})_{2} \mathrm{M}(\mathrm{M}=\mathrm{Ag}, \mathrm{Li}), \mathrm{MEM}-(\mathrm{TCNQ})_{2}$ exihibit a variety of phases. In this work, the microscopic origin of this variety was theoretically searched for with special emphasis on $(\mathrm{TMTCF})_{2} \mathrm{X}$ and $(\mathrm{DI}-\mathrm{DCNQI})_{2} \mathrm{Ag}$.

$(\mathrm{TMTCF})_{2} \mathrm{X}$, where $\mathrm{X}=\mathrm{PF}_{6}, \mathrm{ClO}_{4}, \mathrm{Br}, \mathrm{NO}_{3}$, etc. is a generic name for the compounds $(\mathrm{TMTSF})_{2} \mathrm{X}$, also called Bechgaard salts, and their sulphur analogs (TMTTF) $)_{2} \mathrm{X}$. salts have metallic room temperature resistivity and usually undergo a spin density wave (SDW) transition at $10-12 \mathrm{~K}$ which is caused by the nesting of its Fermi surface. The SDW in (TMTSF $)_{2} \mathrm{PF}_{6}$, for example, has an incommensurate wave number $(0.5,0.20-0.24,0-0.06)$ and an amplitude of $0.08 \mu_{B}$ /molecule 2. 3 . Application of hydrostatic pressure supresses the SDW transition and gives rise to superconductivity. 1 ) In contrast, (TMTTF $)_{2} \mathrm{X}$ salts are semiconducting with a shallow minimum in resistivity at 250-100 K. This paramagnetic insulating phase has been understood as a Mott insulator due to electronic correlation, 0 , Q

\footnotetext{
* E-mail: hseo@watson.phys.s.u-tokyo.ac.jp
} 
band of the TMTCF chain is effectively half-filled because of the dimerization of molecules. The ground states of the members of the (TMTTF $)_{2} \mathrm{X}$ family are either spin-Peierls dimerized or antiferromagnetic states. In the case of $(\mathrm{TMTTF})_{2} \mathrm{Br}$ and $(\mathrm{TMTTF})_{2} \mathrm{SCN}$, the ground state is an AF phase with a commensurate wave number and magnetic moment at ambient pressure of $(1 / 2$, $1 / 4,0)$ and $0.14 \mu_{B} /$ molecule, respectively, as disclosed by Nakamura et al. 8 . 8 ) The line shape observed in these two experiments suggests that the microscopic spin structure in these salts is as shown in Fig. 1.10) Furthermore, in (TMTTF $)_{2} \mathrm{Br}$ under pressure the existence of an AF state with an incommensurate wave number has been indicated 111. This incommensurate phase can be considered to be similar to the SDW phase in (TMTSF $)_{2} \mathrm{X}$.

In order to study the effect of the Coulomb interaction in TMTCF salts, we consider a 1-D chain

Fig. 1. Spin structure of (TMTTF $)_{2} \mathrm{Br}$ and $(\mathrm{TMTTF})_{2} \mathrm{SCN}$ in the TMTTF chain, indicated by NMR experiments. 8. Circles represent TMTTF molecules, and arrows pointing upward (downward) represent places where the up (down) spin appears.

of TMTCF molecules. It is expected that a reasonable description will be provided by the 1-D dimerized extended Hubbard model defined by the Hamiltonian

$$
\begin{aligned}
H= & t_{1} \sum_{i \text { even }, \sigma}\left(a_{i \sigma}^{\dagger} a_{i+1 \sigma}+\text { h.c. }\right)+t_{2} \sum_{i \text { odd }, \sigma}\left(a_{i \sigma}^{\dagger} a_{i+1 \sigma}+\text { h.c. }\right) \\
& +U \sum_{i} n_{i \uparrow} n_{i \downarrow}+V \sum_{i} n_{i} n_{i+1}
\end{aligned}
$$

where $\sigma$ is a spin index and takes $\uparrow$ and $\downarrow, n_{i \sigma}$ and $a_{i \sigma}^{\dagger}\left(a_{i \sigma}\right)$ denote the number operator and the creation (annihilation) operator for the electron of spin $\sigma$ at the $i$ th site and $n_{i}=n_{i \uparrow}+n_{i \downarrow}$. $U$ and $V$ are the on-site and nearest-neighbor Coulomb interactions, respectively.

We treat $U$ and $V$ in the mean field (MF) approximation and follow the treatment by Kino and Fukuyama,12,13 who have elucidated that the variety of ground states of several BEDT-TTF compounds can be understood in terms of the on-site Coulomb interaction and the dimerization as key factors.

We assume that four molecules make the basic lattice periodicity in the chain direction, as is indicated by experiments. Thus there are four different sites in a unit cell and the MF Hamiltonian 
in $k$-space is given by

$$
\begin{aligned}
& H^{M F}=\sum_{k \sigma}\left(\begin{array}{c}
a_{1 k \sigma} \\
a_{2 k \sigma} \\
a_{3 k \sigma} \\
a_{4 k \sigma}
\end{array}\right)^{\dagger}\left[h_{0}+U\left(\begin{array}{cccc}
n_{1 \bar{\sigma}} & & 0 \\
& n_{2 \bar{\sigma}} & & \\
0 & n_{3 \bar{\sigma}} & \\
& & & n_{4 \bar{\sigma}}
\end{array}\right)\right.
\end{aligned}
$$

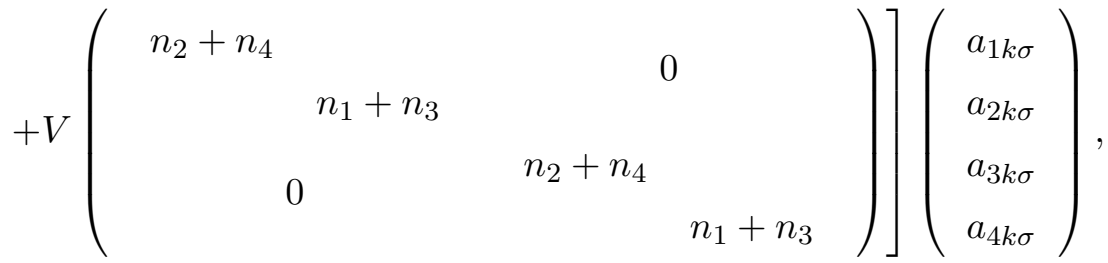

where $\bar{\sigma}$ is opposite to $\sigma$ and $a_{\nu k \sigma}$ is the Fourier transform of $a_{i \sigma}$, i.e. $a_{\nu k \sigma}=\frac{1}{\sqrt{N_{\text {cell }}}} \sum_{\alpha} e^{i R_{\alpha \nu} k} a_{(\alpha \nu) \sigma}$ and $\mathrm{N}_{\text {cell }}$ is the total number of unit cells. $\alpha$ and $\nu$ denote the cell number and the number of the molecule, respectively. The $h_{0}$ term is a matrix which comes from the first term of eq. (1).

Our calculation is carried out at $T=0$ and the electron density is fixed at quarter filling, namely, two electrons per 4 molecules, since the band of (TMTCF $)_{2} \mathrm{X}$ is quarter-filled in terms of holes. It is supposed that the total spin moment in the unit cell is zero, i.e., the solutions we consider are only paramagnetic or antiferromagnetic ones within a chain, and possible ferro/ferrimagnetic states are excluded, which is reasonable based on the results experiments. Actually in the entire range of parameters we show in the following, no ferromagnetic states are stabilized.

In the case of $V=0$, the results indicate that an AF state, whose spin configuration is as shown in the inset of Fig. 2, is stabilized. We set $t_{1} \geq t_{2}$ here, so that the pairs of molecules (1-2) and (3-4) can be considered as dimers. The directions of the spin moments inside the dimers are the same and the AF ordering occurs between dimers, i.e. $\mathrm{S}_{\mathrm{Z}}(1)=\mathrm{S}_{\mathrm{Z}}(2)=-\mathrm{S}_{\mathrm{Z}}(3)=-\mathrm{S}_{\mathrm{Z}}(4)(\uparrow \uparrow \downarrow \downarrow)$. We have plotted the absolute value of spin per molecule, $\mathrm{S}_{Z}$, as a function of $U / t_{2}$ for various values of $t_{1} / t_{2}$ in Fig. 2. It is seen that the magnitude of the spin moment increases rapidly when the on-site Coulomb energy, $U$, increases and that the dependences on $t_{1} / t_{2}$ are not so strong at least in the parameter range investigated. The wave number of this AF state is $2 k_{F}$ which is identical to the 1-D nesting vector, and this AF ordering affects the band structure so as to make the system an insulator. In the small $U$ region where the spin moment is small, the nested Fermi surface SDW picture is valid, while in the large $U$ region Mott AF (between the dimers) picture with larger spin moment would be more suited, although one cannot distinguish these two pictures definitely within the MF approximation. The electron density at each site remains 0.5 when $U$ is varied.

In the presence of finite $V$, a phase transition occurs between two different types of AF states with a fixed value of $U$. We show in Figs. 3 and 4 the spin, $\mathrm{S}_{Z}$, of molecules 1 and 2 for $t_{1} / t_{2}=1.0$ (without dimerization) and 1.1 (with dimerization) with $U / t_{2}$ fixed at 5.0. It is seen that the charge disproportionation, $\delta$, is accompanied by this transition. We note that $\mathrm{S}_{\mathrm{Z}}(1)=-\mathrm{S}_{\mathrm{Z}}(3)$, 
Fig. 2. U-dependence of absolute magnitude of spin moment per molecule, $\mathrm{S}_{Z}$.

$\mathrm{S}_{\mathrm{Z}}(2)=-\mathrm{S}_{\mathrm{Z}}(4)$ and $n_{1}=n_{3}=0.5+\delta, n_{2}=n_{4}=0.5-\delta$, where $n_{i}$ is the electron density at site ' $i$ '.

For comparison, we first analyze the results for $t_{1}=t_{2}$ (Fig. 3), which is the case without dimerization. It is seen that there exists 1st-order transition at $V=V_{c}\left(V_{c} / t_{2} \simeq 0.392\right.$ for $U / t_{2}=$ 5.0) from the $(\uparrow \uparrow \downarrow \downarrow)$ AF state to another AF state, where the spin moment in molecules 2 and 4 is zero and only the up-spin in molecule 1 and the down-spin in the molecule 3 survive ( $\uparrow \downarrow 0$ ), as shown in an inset of Fig. 3. As regards the $V$-dependence of the spin moment, for $V \leq V_{c}$ the absolute value of the moment is not different from that in the case of $V=0$, while for $V \geq V_{c}, \mathrm{~S}_{\mathrm{Z}}(1)=\left|\mathrm{S}_{\mathrm{Z}}(3)\right|$ increases with increasing $V$. This $(\uparrow 0 \downarrow 0)$ AF state is accompanied by the charge disproportionation, i.e. the electrons on molecules 2 and 4 move to molecules 1 and 3. This modulation of the charge is the $4 k_{F}$ charge-density-wave (CDW) whose amplitude is twice the value of $\delta$. This state can be considered as a Wigner crystal.

In the case of $t_{1} \neq t_{2}$ (Fig. 4), i.e. with dimerization, the latter ( $\left.\uparrow \downarrow \downarrow 0\right)$ AF state is modified. There appear spin moments also on molecules 2 and 4 , and the absolute values of the magnetic moments on the two molecules within the dimer are different, as $\mathrm{S}_{Z}(1) \geq \mathrm{S}_{\mathrm{Z}}(2)$ and $\left|\mathrm{S}_{Z}(3)\right| \geq\left|\mathrm{S}_{\mathrm{Z}}(4)\right|$ $(\uparrow \uparrow \downarrow \downarrow)$, which is schematically shown in the inset of Fig. 4. We note that there exists a charge disproportionation with a magnitude similar to that in the case of $t_{1}=t_{2}$. The value of $V=V_{c}$, where this phase transition from the $(\uparrow \uparrow \downarrow \downarrow)$ state to the $(\uparrow \uparrow \downarrow \downarrow)$ state occurs, gets large when one increase the degree of dimerization $t_{1} / t_{2}$, and it appears that the 1st-order phase transition crosses over to a 2 nd-order-like behavior as $t_{1} / t_{2}$ is increased. Above $V_{c}$, the $V$-dependence of the spin moment of molecules 1 and 3 is similar to that in the case of $t_{1}=t_{2}$, though that of molecules 2 and 4 falls rapidly toward zero as $V$ increases. 
Fig. 3. V-dependence of absolute magnitude of spin moment per molecule, $\mathrm{S}_{\mathrm{Z}}$ (a) and charge disproportionation, $\delta$ (b) for $U / t_{2}=5.0, t_{1} / t_{2}=1.0$.

In both cases of $t_{1}=t_{2}$ and $t_{1} \neq t_{2}$, the magnetic moment has a tendency to increase with increasing $U$ when $V$ and $t_{1} / t_{2}$ are fixed.

We now compare the results of the calculation and the experimental results, choosing the parameters of our theoretical model to fit the actual compounds. In (TMTCF) ${ }_{2} \mathrm{X}$, the Coulomb interaction, $U$, on a TMTCF molecule is considered to be almost the same value in both series and of order $1 \mathrm{eV}$. Extended Hückel band calculations 14 15, 16, 17) show that $t_{2} \simeq 0.2 \mathrm{eV}$ for TMTTF compounds and $t_{2} \simeq 0.3 \mathrm{eV}$ for TMTSF compounds. Thus the ratio $U / t_{2} \simeq 5$ for TMTTF is larger than the value $U / t_{2} \simeq 3$ for TMTSF, and the experimental results for the large spin moment observed in (TMTTF $)_{2} \mathrm{Br}$ and $(\mathrm{TMTTF})_{2} \mathrm{SCN}$ compared to the SDW amplitude are consistent with the present theory.

The value of $t_{1} / t_{2}$ is expected to be about 1.1 in both (TMTTF) ${ }_{2} \mathrm{Br}$ and (TMTTF) ${ }_{2} \mathrm{SCN}$, 14, 15, (6, 17) hence Fig. 4 applies. The spin structure $(\uparrow 0 \downarrow 0)$ suggested by the results of H-NMR experiments8.60) in (TMTTF $)_{2} \mathrm{Br}$ and (TMTTF $)_{2} \mathrm{SCN}$, which is shown in Fig. 1, is apparently different from that given in Fig. 4, but this apparent discrepancy can be understood as follows. When the $(\uparrow \uparrow \downarrow \downarrow)$ AF state is realized and the spin moment in molecules 2 and 4 is negligible 
Fig. 4. V-dependence of absolute magnitude of spin moment per molecule, $\mathrm{S}_{\mathrm{Z}}$ (a) and charge transfer, $\delta$ (b) for $U / t_{2}=5.0, t_{1} / t_{2}=1.1$

compared to that in molecules 1 and 3, i.e. when $S_{Z}(2)=\left|S_{Z}(4)\right| \ll S_{Z}(1)=\left|S_{Z}(3)\right|$, the observed line shape will be indistinguishable from that in the case of $(\uparrow 0 \downarrow 0)$. It is seen in Fig. 4 that $\mathrm{S}_{Z}(2) \lesssim 0.1 \times \mathrm{S}_{Z}(1)$ when $V / U \gtrsim 0.25$. Such values of $V / U$ are consistent with the estimate by Mila, 18 and the quantum chemistry calculations. 19,200 Consequently we conclude that the long-range Coulomb interaction plays an essential role in (TMTTF $)_{2} \mathrm{Br}$ and $(\mathrm{TMTTF})_{2} \mathrm{SCN}$, and probably in the other TMTTF series as well.

The $(\uparrow \uparrow \downarrow \downarrow)$ state is also possible in the presence of two-fold periodic potential, instead of the nearest-neighbor Coulomb interaction. This case with two-fold periodic potential has been investigated in another context.21 It turned out, however, that to realize a state without $V$ where the spin moment in molecules 2 and 4 is negligible compared to that in molecules 1 and 3, we need to introduce an unreasonably large two-fold periodic potential.22) Furthermore, the existence of such two-fold periodic potential is not expected from the crystallography, 10 23 because the anions are located at the vertices of a parallelipiped and two TMTCF molecules are placed centrosymmetrically in the central cavities.

Next we will analyze the effect of pressure on (TMTCF $)_{2} \mathrm{X}$. It is considered that the bandwidth 
increases and the degree of dimerization decreases with increasing pressure, while $U$ will be hardly affected, then the reduced on-site Coulomb interaction $U / t_{2}$ and the ratio $t_{1} / t_{2}$ will both decrease. The present theoretical results indicate that the magnetic moment decreases when $U / t_{2}$ and/or $t_{1} / t_{2}$ decreases. Thus it is expected that the materials whose ground state is Mott AF at ambient pressure will be transformed into an SDW as the pressure is increased. In (TMTTF) ${ }_{2} \mathrm{Br}$ the transition between a Mott AF phase to an SDW phase was actually observed.11)

Finally we discuss the properties of (DCNQI $)_{2} \mathrm{Ag}$, which is quite similar to (TMTTF $)_{2} \mathrm{X}$. A recent resistivity measurement24) on (DI-DCNQI) ${ }_{2} \mathrm{Ag}$ has disclosed an insulating behavior in the entire temperature range below $300 \mathrm{~K}$. Below $200 \mathrm{~K}$, a $4 k_{F}$ CDW which is of the charge modulation type has been observed in an NMR experiment, 25) and an AF transition occurs at $5.5 \mathrm{~K}$.24) On the other hand, (DMe-DCNQI) ${ }_{2} \mathrm{Ag}$ is metallic down to $100 \mathrm{~K}$, below which the system crosses over to insulating phase accompanied by a $4 k_{F} \mathrm{CDW}$, as suggested by x-ray diffusive scattering.26)

Since $(\mathrm{DCNQI})_{2} \mathrm{Ag}$ systems have no dimerization along the DCNQI stack direction, i.e. $t_{1}=$ $t_{2}\left(=t_{\|}\right)$, in contrast to the TMTCF family, which is quarter-filled, the existence of a Mott insulator produced by on-site Coulomb interaction, $U$, is not possible, while a Wigner crystal due to nearestneighbor Coulomb interaction, $V$, is a candidate. Actually, the intrachain transfer energy $t_{\|}$is estimated to be about $0.2 \mathrm{eV}$ by first principles calculation,27) and the on-site Coulomb interaction $U$ is of the order of $1 \mathrm{eV}$, so $U / t_{\|} \simeq 5$, which is similar to the value for TMTTF salts. Moreover, $V$ is expected to be quite large as in the case of TMTTF compounds. Then the electronic structure of the ground state is expected to be $(\uparrow 0 \downarrow 0)$ AF state as shown in Fig. 3. This consideration is consistent with the observed $4 k_{F}$ CDW 25, 26)

We note here that, in spite of the capability of the exploration of the general trends in a unified way so far demonstrated, the present MF scheme does not take account of fluctuations, and overestimates the stability of magnetic orderings. In fact, the magnetic moments obtained in our MF calculations are larger than the observed value in TMTCF compounds. For understanding of such more quantitative features, effects of the interchain transfer should be taken into account, which is under study.

In summary, we have studied the effects of on-site and nearest-neighbor Coulomb interaction in a quarter-filled 1-D band with and without dimerization, and found that various types of AF spin structure are stabilized. In the absence of $V$, the spin structure of the type $(\uparrow \uparrow \downarrow \downarrow)$ is the ground state independent of the degree of the dimerization, and the charge density is uniform. The staggered magnetization is larger for larger $U$ in general. On the other hand, once $V$ is larger than some critical value charge disproportionation is realized which can be considered as a tendency toward formation of the Wigner crystal. The spin structure in this case is $(\uparrow 0 \downarrow 0)$ or $(\uparrow \uparrow \downarrow \downarrow)$ depending on without or with dimerization. These theoretical results explain experimental findings in $(\mathrm{TMTTF})_{2} \mathrm{X}$ and predict the $\mathrm{AF}$ spin structure in (DI-DCNQI $)_{2} \mathrm{Ag}$. This is the first reported 
study which has clarified the unique roles of $V$ and dimerization in stabilization of characteristic spin structures in 1-D quarter-filled band.

\section{Acknowledgements}

We thank H. Kino, T. Takahashi, S. Kagoshima, K. Hiraki, K. Kanoda, D. Yoshioka and H. Kohno for useful discussions and suggestions. This work was financially supported by a Grant-inAid for Scientific Research on Priority Area "Anomalous Metallic State near the Mott Transition" (07237102) from the Ministry of Education, Science, Sports and Culture.

[1] For review, see D. Jérome: Organic Conductors, ed. J. P. Farges (M. Dekker, New York, 1994).

[2] T. Takahashi, Y. Maniwa, H. Kawamura and G. Saito: J. Phys. Soc. Jpn. 55 (1986) 1364.

[3] J. M. Delrieu, M. Roger, Z. Toffano, A. Mopadour and K. Bechgaard: J. Physique 47 (1986) 839.

[4] D. Jérome, A. Mazaud, M. Ribault and K. Bechgaard: J. Physique Lett. 41 (1980) L95.

[5] S. Barisic and S. Brazovskii: Recent Developments in Condensed Matter Physics, ed. J. Deversee (Plenum, New York, 1981) Vol.1.

[6] V. J. Emery, R. Bruinsma and S. Barisic: Phys. Rev. Lett. 48 (1982) 1039.

[7] J. B. Torrance: J. Physique Colloq. C3 (1983) 799.

[8] T. Nakamura, T. Nobutoki, Y. Kobayashi, T. Takahashi and G. Saito: Synth. Met. 70 (1995) 1293.

[9] T. Nakamura, R. Kinami, T. Takahashi and G. Saito: to be published in Synth. Met.

[10] T. Takahashi: private communications.

[11] B. J. Klemme, S. E. Brown, P. Wzietek, G. Kriza, P. Batail, D. Jérome and J. M. Fabre: Phys. Rev. Lett. 75 (1995) 2408.

[12] H. Kino and H. Fukuyama: J. Phys. Soc. Jpn. 64 (1995) 1877; 64 (1995) 2726; 64 (1995) 4523.

[13] H. Kino and H. Fukuyama: J. Phys. Soc. Jpn. 65 (1996) 2158.

[14] T. Mori, A. Kobayashi, Y. Sasaki and H. Kobayashi: Chem. Lett. (1982) 1923.

[15] P. M. Grant: J. Physique Colloq. C3 (1983) 847.

[16] T. Mori, A. Kobayashi, Y. Sasaki, H. Kobayashi, G. Saito and H. Inokuchi: Bull. Chem. Soc. Jpn. 57 (1984) 627.

[17] L. Ducasse, M. Abderrabba, J. Hoarau, M. Pesquer, B. Gallois and J. Gaultier: J. Phys. C 19 (1986) 3805.

[18] F. Mila: Phys. Rev. B 52 (1995) 4788.

[19] A. Fritsch and L. Ducasse: J. Physique I 1 (1991) 855.

[20] F. Castet, A. Fritsch and L. Ducasse: J. Physique I 6 (1996) 583.

[21] N. Tanemura and Y. Suzumura: J. Phys. Soc. Jpn. 65 (1996) 1792.

[22] H. Seo and H. Fukuyama: to be published.

[23] S. Kagoshima: private communications.

[24] K. Hiraki and K. Kanoda: Phys. Rev. B 54 (1996) 17276.

[25] K. Hiraki and K. Kanoda: private communications.

[26] R. Moret, P. Erk, S. Hünig and J. U. Von Shültz: J. Physique 49 (1988) 1925.

[27] T. Miyazaki, K. Terakura, Y. Morikawa and T. Yamasaki: Phys. Rev. B 54 (1996) 10452. 


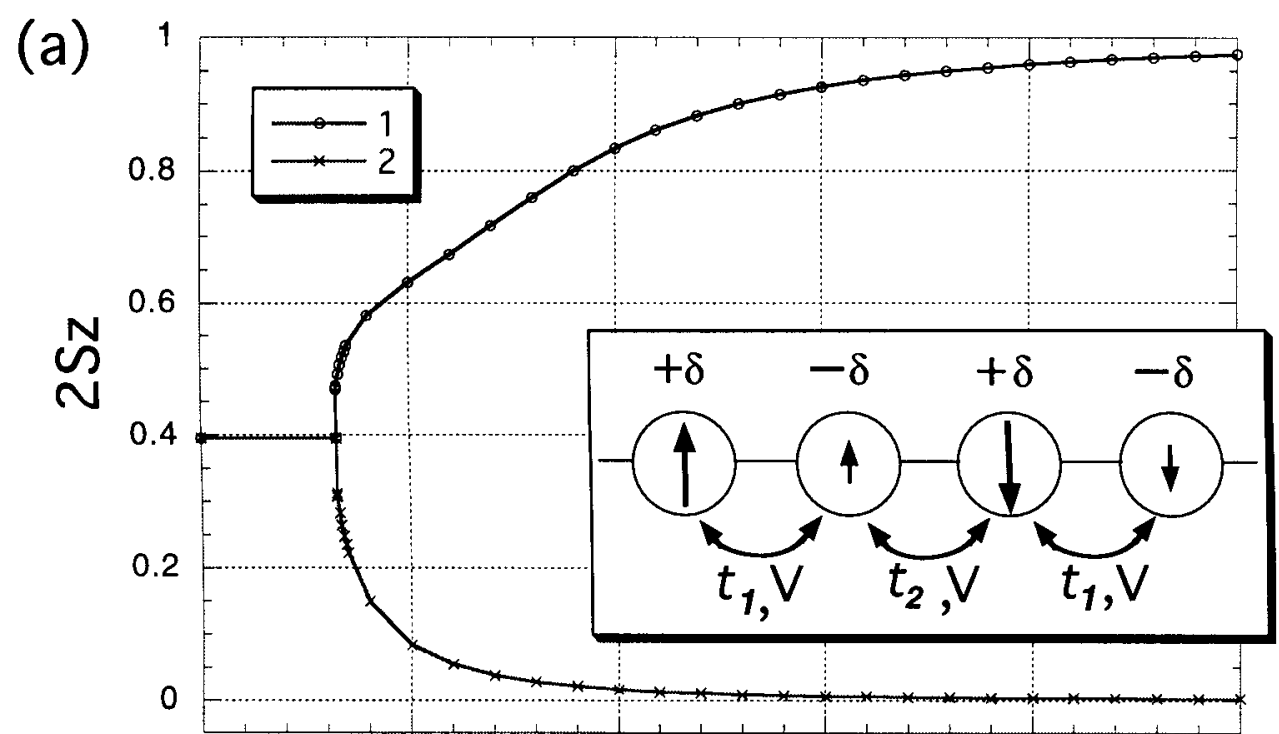

(b)

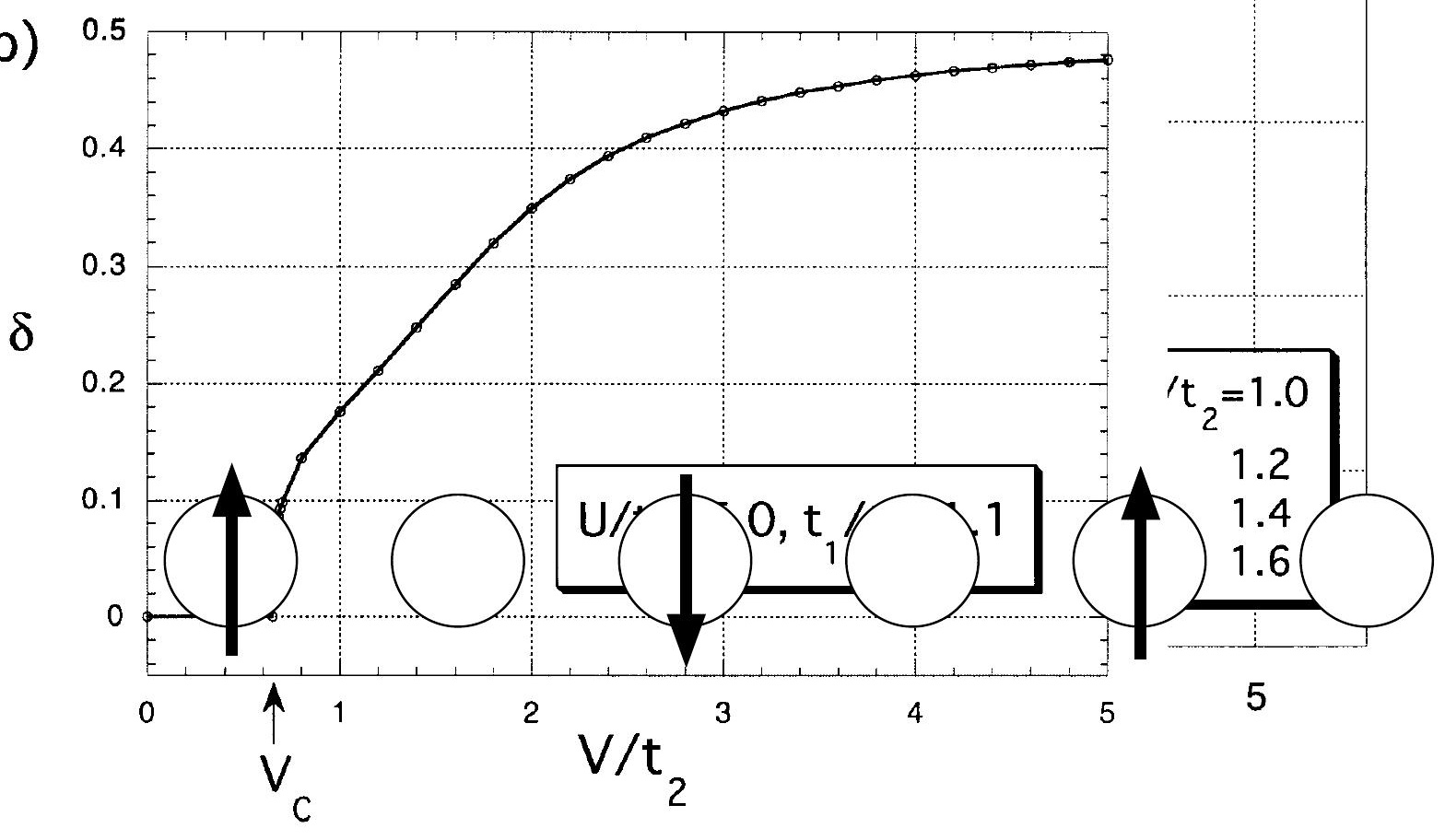

